\title{
Multiple solutions of a $p$-Laplacian model involving a fractional derivative
}

\author{
Xiping Liu ${ }^{1 *}$, Mei Jia ${ }^{1 *}$ and Weigao $\mathrm{Ge}^{2}$
}

\section{"Correspondence:}

xipingliu@usst.edu.cn;

jiamei-usst@163.com

${ }^{1}$ College of Science, University of

Shanghai for Science and

Technology, Shanghai, 200093,

China

Full list of author information is

available at the end of the article

\begin{abstract}
In this paper, we study the $p$-Laplacian model involving the Caputo fractional derivative with Dirichlet-Neumann boundary conditions. Using a fixed point theorem, we prove the existence of at least three solutions of the model. As an application, an example is included to illustrate the main results.
\end{abstract}

\section{Introduction}

In this paper, we are concerned with the multiple positive solutions of Dirichlet-Neumann boundary value problems for a type of fractional differential equation involving a $p$-Laplacian operator as the following form:

$$
\left\{\begin{array}{l}
\left(\varphi_{p}\left({ }^{C} D^{\alpha} x(t)\right)\right)^{\prime}=\varphi_{p}(\lambda) f\left(t, x(t), x^{\prime}(t)\right), \quad t \in(0,1), \\
k_{0} x(0)-k_{1} x(1)=0, \\
m_{0} x^{\prime}(0)-m_{1} x^{\prime}(1)=0, \\
x^{(r)}(0)=0, \quad r=2,3, \ldots, n,
\end{array}\right.
$$

where $\varphi_{p}$ is the $p$-Laplacian operator, i.e., $\varphi_{p}(s)=|s|^{p-2} s, p>1$, and $\varphi_{q}=\varphi_{p}^{-1}, \frac{1}{p}+\frac{1}{q}=1$; ${ }^{C} D^{\alpha}$ is the standard Caputo derivative; $2 \leq n-1<\alpha \leq n$, and $n$ is an integer; $0<\lambda \in \mathbb{R}, k_{i}, m_{i}$ are constants, $i=0,1 ; f$ is a given function.

It is well known that both the fractional differential equations and the $p$-Laplacian operator equations are widely used in the fields of different physical and natural phenomena, non-Newtonian mechanics, nonlinear elasticity and glaciology, combustion theory, population biology, complex geometry and patterns. Many researchers have extensively studied either the fractional differential equations or the $p$-Laplacian operator equations, respectively. For details of the theory and applications of the fractional differential equations or the $p$-Laplacian operator equations, see [1-15] and the references therein.

The authors of [13] studied the boundary value problem of fractional order

$$
\left\{\begin{array}{l}
{ }^{C} D^{\alpha} u(t)=f\left(t, u(t), u^{\prime}(t)\right), \quad t \in(0,1), 1<\alpha \leq 2, \\
u(0)+u^{\prime}(0)=0 \\
u(1)+u^{\prime}(1)=0 .
\end{array}\right.
$$

By means of the Schauder fixed point theorem and an extension of the Krasnosel'skii fixed point theorem in a cone, the existence of positive solutions is obtained.

() 2013 Liu et al.; licensee Springer. This is an Open Access article distributed under the terms of the Creative Commons Attribution License (http://creativecommons.org/licenses/by/2.0), which permits unrestricted use, distribution, and reproduction in any medium, provided the original work is properly cited. 
In [14], the authors investigated the nonlinear boundary value problem of fractional differential equation

$$
\left\{\begin{array}{l}
{ }^{C} D^{\alpha} x(t)=f\left(t, x(t), x^{\prime}(t)\right), \quad t \in(0,1), \\
g_{0}\left(x(0), x^{\prime}(0)\right)=0, \\
g_{1}\left(x(1), x^{\prime}(1)\right)=0, \\
x^{\prime \prime}(0)=x^{\prime \prime \prime}(0)=\cdots=x^{(n)}=0 .
\end{array}\right.
$$

By means of the Amann theorem and the method of upper and lower solutions, some results on the multiple solutions are obtained.

Liu [15] was concerned with the mixed type multi-point boundary value problem

$$
\left\{\begin{array}{l}
\left(\varphi_{p}\left(x^{\prime}(t)\right)\right)^{\prime}=-f\left(t, x(t), x^{\prime}(t)\right), \quad t \in(0,1), \\
x(0)-\alpha x^{\prime}(0)=\lambda_{1}, \\
x(1)-\sum_{i=1}^{m} \beta_{i} x\left(\xi_{i}\right)=\lambda_{2},
\end{array}\right.
$$

the existence of at least three positive solutions of the above mentioned boundary value problem is established.

Recently, a few researchers were devoted to the study of boundary value problems for the fractional differential equations with the $p$-Laplacian operator equations (see [16, 17]). In [16], some results on the existence and uniqueness of a solution for the following boundary value problem of a fractional differential equation are obtained:

$$
\left\{\begin{array}{l}
\left(\varphi_{p}\left({ }^{C} D^{\alpha} x(t)\right)\right)^{\prime}=f(t, x(t)), \quad t \in(0,1) \\
x(0)=r_{0} x(1) \\
x^{\prime}(0)=r_{1} x^{\prime}(1) .
\end{array}\right.
$$

Motivated by the above, the purpose of this paper is to establish the existence of multiple positive solutions to boundary value problems for a fractional differential equation involving a $p$-Laplacian operator (1). If $p$ is an integer, the equation in (1) reduces to a standard nonlinear fractional differential equation. And it will become a standard $p$-Laplacian operator equation when $\alpha$ is an integer. Therefore, our results in this paper are the promotion and more general case of these two types of problems. By means of the fixed point theorem due to Avery and Peterson, we prove the results that there exist at least three positive solutions of the boundary value problem (1). As an application, an example is included to illustrate the main results.

\section{Preliminaries}

In this section, we give the definition of a fractional derivative and some lemmas, which will be used later.

Definition 2.1 [18] Let $\alpha>0$ for a function $y:(0,+\infty) \rightarrow \mathbb{R}$. The fractional integral of order $\alpha$ of $y$ is defined by

$$
I^{\alpha} y(t)=\frac{1}{\Gamma(\alpha)} \int_{0}^{t}(t-s)^{\alpha-1} y(s) \mathrm{d} s,
$$


provided the integral exists. The Caputo derivative of a function $y:(0,+\infty) \rightarrow \mathbb{R}$ is given by

$$
{ }^{C} D^{\alpha} y(t)=\frac{1}{\Gamma(n-\alpha)} \int_{0}^{t} \frac{y^{(n)}(s)}{(t-s)^{\alpha+1-n}} \mathrm{~d} s
$$

provided the right-hand side is pointwise defined on $(0,+\infty)$, where $n$ is an integer, with $n=[\alpha]+1$.

$\Gamma$ denotes the gamma function, that is,

$$
\Gamma(\alpha)=\int_{0}^{+\infty} e^{-t} t^{\alpha-1} \mathrm{~d} t
$$

From Definition 2.1, we can obtain the following lemma.

Lemma 2.1 Let $0<n-1<\alpha \leq n$. If we assume $y \in C^{n}(0,1) \cap L[0,1]$, the fractional differential equation

$$
{ }^{C} D^{\alpha} y(t)=0
$$

has a unique solution

$$
y(t)=\sum_{k=0}^{n-1} \frac{y^{(k)}(0)}{k !} t^{k}
$$

Throughout this paper, we always suppose the following condition holds.

(H0) The parameters in the boundary value problem (1) satisfy the following conditions:

$$
2 \leq n-1<\alpha \leq n, \quad 0<k_{1}<k_{0}, \quad 0<m_{1}<m_{0} .
$$

Lemma 2.2 Suppose that $(\mathrm{H} 0)$ holds and $h \in C[0,1]$. Then the boundary value problem

$$
\left\{\begin{array}{l}
\left(\varphi_{p}\left({ }^{C} D^{\alpha} x(t)\right)\right)^{\prime}=\varphi_{p}(\lambda) h(t), \quad t \in(0,1), \\
k_{0} x(0)-k_{1} x(1)=0, \\
m_{0} x^{\prime}(0)-m_{1} x^{\prime}(1)=0, \\
x^{(r)}(0)=0, \quad r=2,3, \ldots, n
\end{array}\right.
$$

has a unique solution

$$
\begin{aligned}
x(t)= & \frac{m_{1}}{m_{0}-m_{1}} \cdot \frac{\lambda}{\Gamma(\alpha-1)} \cdot\left(\frac{k_{1}}{k_{0}-k_{1}}+t\right) \int_{0}^{1}(1-\tau)^{\alpha-2} \varphi_{q}\left(\int_{0}^{\tau} h(s) \mathrm{d} s\right) \mathrm{d} \tau \\
& +\frac{k_{1}}{k_{0}-k_{1}} \cdot \frac{\lambda}{\Gamma(\alpha)} \int_{0}^{1}(1-\tau)^{\alpha-1} \varphi_{q}\left(\int_{0}^{\tau} h(s) \mathrm{d} s\right) \mathrm{d} \tau \\
& +\frac{\lambda}{\Gamma(\alpha)} \int_{0}^{t}(t-\tau)^{\alpha-1} \varphi_{q}\left(\int_{0}^{\tau} h(s) \mathrm{d} s\right) \mathrm{d} \tau .
\end{aligned}
$$

That is, every solution of (2) is also a solution of (3) and vice versa. 
Proof The definition of the Caputo derivative implies that ${ }^{C} D^{\alpha} x(0)=0$, and from (2) we have

$$
\varphi_{p}\left({ }^{C} D^{\alpha} x(t)\right)=\varphi_{p}(\lambda) \int_{0}^{t} h(s) \mathrm{d} s, \quad \text { and } \quad{ }^{C} D^{\alpha} x(t)=\lambda \varphi_{q}\left(\int_{0}^{t} h(s) \mathrm{d} s\right) .
$$

So,

$$
\begin{aligned}
x(t) & =x(0)+x^{\prime}(0) t+\frac{x^{\prime \prime}(0)}{2 !} t^{2}+\cdots+\frac{x^{(n-1)}(0)}{(n-1) !} t^{n-1}+I^{\alpha} \lambda \varphi_{q}\left(\int_{0}^{t} h(s) \mathrm{d} s\right) \\
& =x(0)+x^{\prime}(0) t+\frac{\lambda}{\Gamma(\alpha)} \int_{0}^{t}(t-\tau)^{\alpha-1} \varphi_{q}\left(\int_{0}^{\tau} h(s) \mathrm{d} s\right) \mathrm{d} \tau .
\end{aligned}
$$

By using the property of the fractional derivatives and integrals, we can get

$$
x^{\prime}(t)=x^{\prime}(0)+\frac{\lambda}{\Gamma(\alpha-1)} \int_{0}^{t}(t-\tau)^{\alpha-2} \varphi_{q}\left(\int_{0}^{\tau} h(s) \mathrm{d} s\right) \mathrm{d} \tau .
$$

Then

$$
\begin{aligned}
& x(1)=x(0)+x^{\prime}(0)+\frac{\lambda}{\Gamma(\alpha)} \int_{0}^{1}(1-\tau)^{\alpha-1} \varphi_{q}\left(\int_{0}^{\tau} h(s) \mathrm{d} s\right) \mathrm{d} \tau, \\
& x^{\prime}(1)=x^{\prime}(0)+\frac{\lambda}{\Gamma(\alpha-1)} \int_{0}^{1}(1-\tau)^{\alpha-2} \varphi_{q}\left(\int_{0}^{\tau} h(s) \mathrm{d} s\right) \mathrm{d} \tau .
\end{aligned}
$$

From the boundary condition $k_{0} x(0)-k_{1} x(1)=0$, and $m_{0} x^{\prime}(0)-m_{1} x^{\prime}(1)=0$, we can obtain that

$$
\begin{aligned}
x(0)= & \frac{k_{1}}{k_{0}-k_{1}}\left(\frac{m_{1}}{m_{0}-m_{1}} \cdot \frac{\lambda}{\Gamma(\alpha-1)} \cdot \int_{0}^{1}(1-\tau)^{\alpha-2} \varphi_{q}\left(\int_{0}^{\tau} h(s) \mathrm{d} s\right) \mathrm{d} \tau\right. \\
& \left.+\frac{\lambda}{\Gamma(\alpha)} \cdot \int_{0}^{1}(1-\tau)^{\alpha-1} \varphi_{q}\left(\int_{0}^{\tau} h(s) \mathrm{d} s\right) \mathrm{d} \tau\right), \\
x^{\prime}(0)= & \frac{m_{1}}{m_{0}-m_{1}} \cdot \frac{\lambda}{\Gamma(\alpha-1)} \cdot \int_{0}^{1}(1-\tau)^{\alpha-2} \varphi_{q}\left(\int_{0}^{\tau} h(s) \mathrm{d} s\right) \mathrm{d} \tau .
\end{aligned}
$$

Substituting (5) and (6) into (4), we can obtain that

$$
\begin{aligned}
x(t)= & \frac{m_{1}}{m_{0}-m_{1}} \cdot \frac{\lambda}{\Gamma(\alpha-1)} \cdot\left(\frac{k_{1}}{k_{0}-k_{1}}+t\right) \int_{0}^{1}(1-\tau)^{\alpha-2} \varphi_{q}\left(\int_{0}^{\tau} h(s) \mathrm{d} s\right) \mathrm{d} \tau \\
& +\frac{k_{1}}{k_{0}-k_{1}} \cdot \frac{\lambda}{\Gamma(\alpha)} \int_{0}^{1}(1-\tau)^{\alpha-1} \varphi_{q}\left(\int_{0}^{\tau} h(s) \mathrm{d} s\right) \mathrm{d} \tau \\
& +\frac{\lambda}{\Gamma(\alpha)} \int_{0}^{t}(t-\tau)^{\alpha-1} \varphi_{q}\left(\int_{0}^{\tau} h(s) \mathrm{d} s\right) \mathrm{d} \tau .
\end{aligned}
$$

The proof is completed. 
Let $E=C^{1}[0,1],\|x\|=\max \left\{\max _{t \in[0,1]}|x(t)|, \max _{t \in[0,1]}\left|x^{\prime}(t)\right|\right\}$, then $(E,\|\cdot\|)$ is a Banach space. Set

$$
\begin{aligned}
P= & \left\{x \in E\left|x(t) \geq 0, \max _{t \in[0,1]}\right| x(t) \mid \leq \min \left\{\frac{k_{0}}{k_{0}-k_{1}} \max _{t \in[0,1]}\left|x^{\prime}(t)\right|, \frac{k_{0}}{k_{1}} \min _{t \in[0,1]}|x(t)|\right\},\right. \\
& x \text { is monotone increasing and convex on }[0,1]\},
\end{aligned}
$$

then $P$ is a cone on $E$.

Define the operator $T: P \rightarrow E$ by

$$
\begin{aligned}
& (T x)(t) \\
& =\frac{m_{1}}{m_{0}-m_{1}} \cdot \frac{\lambda}{\Gamma(\alpha-1)} \cdot\left(\frac{k_{1}}{k_{0}-k_{1}}+t\right) \int_{0}^{1}(1-\tau)^{\alpha-2} \varphi_{q}\left(\int_{0}^{\tau} f\left(s, x(s), x^{\prime}(s)\right) \mathrm{d} s\right) \mathrm{d} \tau \\
& \quad+\frac{k_{1}}{k_{0}-k_{1}} \cdot \frac{\lambda}{\Gamma(\alpha)} \int_{0}^{1}(1-\tau)^{\alpha-1} \varphi_{q}\left(\int_{0}^{\tau} f\left(s, x(s), x^{\prime}(s)\right) \mathrm{d} s\right) \mathrm{d} \tau \\
& \quad+\frac{\lambda}{\Gamma(\alpha)} \int_{0}^{t}(t-\tau)^{\alpha-1} \varphi_{q}\left(\int_{0}^{\tau} f\left(s, x(s), x^{\prime}(s)\right) \mathrm{d} s\right) \mathrm{d} \tau .
\end{aligned}
$$

It is clear that $x \in C^{n}[0,1]$ is the solution of the boundary value problem (1) if and only if $x \in E$ is the fixed point of the operator $T$.

Lemma 2.3 Suppose that (H0) holds and the function $f \in C([0,1] \times[0,+\infty) \times \mathbb{R},[0,+\infty)$ ). Then $T: P \rightarrow P$ is completely continuous.

Proof By the definition of the operator $T$, it is easy to see $(T x)(t) \geq 0$ for any $t \in[0,1]$. And using the property of the fractional integrals and derivatives, we can get that

$$
\begin{aligned}
(T x)^{\prime}(t)= & \frac{m_{1}}{m_{0}-m_{1}} \cdot \frac{\lambda}{\Gamma(\alpha-1)} \int_{0}^{1}(1-\tau)^{\alpha-2} \varphi_{q}\left(\int_{0}^{\tau} f\left(s, x(s), x^{\prime}(s)\right) \mathrm{d} s\right) \mathrm{d} \tau \\
& +\frac{\lambda}{\Gamma(\alpha-1)} \int_{0}^{t}(t-\tau)^{\alpha-2} \varphi_{q}\left(\int_{0}^{\tau} f\left(s, x(s), x^{\prime}(s)\right) \mathrm{d} s\right) \mathrm{d} \tau \\
\geq & 0 .
\end{aligned}
$$

And

$$
(T x)^{\prime \prime}(t)=\frac{\lambda}{\Gamma(\alpha-2)} \int_{0}^{t}(t-\tau)^{\alpha-3} \varphi_{q}\left(\int_{0}^{\tau} f\left(s, x(s), x^{\prime}(s)\right) \mathrm{d} s\right) \mathrm{d} \tau \geq 0 .
$$

Then $T x$ is nonnegative, monotone increasing and convex on $[0,1]$. They imply that

$$
\begin{aligned}
& \max _{t \in[0,1]}|(T x)(t)|=\max _{t \in[0,1]}(T x)(t)=(T x)(1), \\
& \max _{t \in[0,1]}\left|(T x)^{\prime}(t)\right|=\max _{t \in[0,1]}(T x)^{\prime}(t)=(T x)^{\prime}(1),
\end{aligned}
$$

and

$$
\min _{t \in[0,1]}(T x)(t)=(T x)(0), \quad \min _{t \in[0,1]}(T x)^{\prime}(t)=(T x)^{\prime}(0) .
$$


Therefore

$$
\max _{t \in[0,1]}|(T x)(t)|=(T x)(1)=\frac{k_{0}}{k_{1}}(T x)(0)=\frac{k_{0}}{k_{1}} \min _{t \in[0,1]}|(T x)(t)| .
$$

And

$$
\begin{aligned}
& \max _{t \in[0,1]}|(T x)(t)| \\
&=(T x)(1) \\
&=\frac{m_{1}}{m_{0}-m_{1}} \cdot \frac{k_{0}}{k_{0}-k_{1}} \cdot \frac{\lambda}{\Gamma(\alpha-1)} \int_{0}^{1}(1-\tau)^{\alpha-2} \varphi_{q}\left(\int_{0}^{\tau} f\left(s, x(s), x^{\prime}(s)\right) \mathrm{d} s\right) \mathrm{d} \tau \\
&+\frac{k_{0}}{k_{0}-k_{1}} \cdot \frac{\lambda}{\Gamma(\alpha)} \int_{0}^{1}(1-\tau)^{\alpha-1} \varphi_{q}\left(\int_{0}^{\tau} f\left(s, x(s), x^{\prime}(s)\right) \mathrm{d} s\right) \mathrm{d} \tau \\
& \leq \frac{m_{1}}{m_{0}-m_{1}} \cdot \frac{k_{0}}{k_{0}-k_{1}} \cdot \frac{\lambda}{\Gamma(\alpha-1)} \int_{0}^{1}(1-\tau)^{\alpha-2} \varphi_{q}\left(\int_{0}^{\tau} f\left(s, x(s), x^{\prime}(s)\right) \mathrm{d} s\right) \mathrm{d} \tau \\
&+\frac{k_{0}}{k_{0}-k_{1}} \cdot \frac{\lambda}{\Gamma(\alpha-1)} \int_{0}^{1}(1-\tau)^{\alpha-2} \varphi_{q}\left(\int_{0}^{\tau} f\left(s, x(s), x^{\prime}(s)\right) \mathrm{d} s\right) \mathrm{d} \tau \\
&= \frac{k_{0}}{k_{0}-k_{1}} \cdot \frac{m_{0}}{m_{0}-m_{1}} \cdot \frac{\lambda}{\Gamma(\alpha-1)} \int_{0}^{1}(1-\tau)^{\alpha-2} \varphi_{q}\left(\int_{0}^{\tau} f\left(s, x(s), x^{\prime}(s)\right) \mathrm{d} s\right) \mathrm{d} \tau \\
&= \frac{k_{0}}{k_{0}-k_{1}} \cdot(T x)^{\prime}(1) \\
&= \frac{k_{0}}{k_{0}-k_{1}} \max _{t \in[0,1]}\left|(T x)^{\prime}(t)\right| .
\end{aligned}
$$

Then

$$
\max _{t \in[0,1]}|(T x)(t)| \leq \min \left\{\frac{k_{0}}{k_{0}-k_{1}} \max _{t \in[0,1]}\left|(T x)^{\prime}(t)\right|, \frac{k_{0}}{k_{1}} \min _{t \in[0,1]}|(T x)(t)|\right\} .
$$

Thus, $T x \in P$, so $T: P \rightarrow P$.

It is easy to prove that $T$ is continuous and compact if the conditions of the lemma hold. The proof is complete.

For convenience of the readers, we provide some background material from the theory of cones in Banach spaces and the Avery-Peterson fixed point theorem.

Definition 2.2 Let $E$ be a Banach space and let $P \subset E$ be a cone. A continuous map $\gamma$ is called a concave (resp. convex) functional on $P$ if and only if $\gamma(t x+(1-t) y) \geq t \gamma(x)+(1-$ $t) \gamma(y)($ resp. $\gamma(t x+(1-t) y) \leq t \gamma(x)+(1-t) \gamma(y))$ for all $x, y \in P$ and $0<t<1$.

Let $\beta$ and $\rho$ be nonnegative continuous convex functionals on a cone $P$, let $\omega$ be a nonnegative continuous concave functional on a cone $P$, and let $\psi$ be a nonnegative continuous functional on a cone $P$. Then, for positive real numbers $a, b, c$ and $d$, we define the following convex sets:

$$
\begin{aligned}
& P(\beta ; d)=\{x \in P \mid \beta(x)<d\}, \quad \bar{P}(\beta ; d)=\{x \in P \mid \beta(x) \leq d\} ; \\
& P(\beta, \omega ; b, d)=\{x \in P \mid \beta(x) \leq d, \omega(x) \geq b\}
\end{aligned}
$$




$$
\begin{aligned}
& P(\beta, \rho, \omega ; b, c, d)=\{x \in P \mid \beta(x) \leq d, \rho(x) \leq c, \omega(x) \geq b\} ; \\
& R(\beta, \psi ; a, d)=\{x \in P \mid \beta(x) \leq d, \psi(x) \geq a\} .
\end{aligned}
$$

Lemma 2.4 (Avery-Peterson fixed point theorem [19]) Let P be a cone in a real Banach space $E$. Let $\beta$ and $\rho$ be nonnegative continuous convex functionals on $P$, let $\omega$ be a nonnegative continuous concave functional on $P$, and let $\psi$ be a nonnegative continuous functional on $P$ satisfying $\psi(k x) \leq k \psi(x)$ for $0<k<1$, such that for some positive numbers $M$ and $d$, $\omega(x) \leq \psi(x)$ and $\|x\| \leq M \beta(x)$ for all $x \in \bar{P}(\beta, d)$.

Suppose that $T: \bar{P}(\beta ; d) \rightarrow \bar{P}(\beta ; d)$ is completely continuous and there exist positive numbers $a, b$ and $c$ with $a<b$ such that

(A1) $\{x \in P(\beta, \rho, \omega ; b, c, d) \mid \omega(x)>b\} \neq \phi$, and $\omega(T x)>b$ for all $x \in P(\beta, \rho, \omega ; b, c, d)$;

(A2) $\omega(T x)>b$ for all $x \in P(\beta, \omega ; b, d)$ with $\rho(T x)>c$;

(A3) $0 \notin R(\beta, \psi ; a, d)$ and $\psi(T x)<a$ for $x \in R(\beta, \psi ; a, d)$ with $\psi(x)=a$.

Then $T$ has at least three fixed points $x_{1}, x_{2}, x_{3} \in \bar{P}(\beta ; d)$ such that $\beta\left(x_{i}\right)<d, i=1,2,3$; $\omega\left(x_{1}\right)>b ; \psi\left(x_{2}\right)>a$, with $\omega\left(x_{2}\right)<b$; and $\psi\left(x_{3}\right)<a$.

\section{Multiple positive solutions of the boundary value problems}

In this section, we establish the existence of multiple positive solutions of the boundary value problem (1).

Denote

$$
M=\frac{k_{0}}{k_{0}-k_{1}}, \quad r_{0}=\frac{k_{1}}{k_{0}-k_{1}} \cdot \frac{\Gamma(\alpha+q-1)}{\Gamma(\alpha+q)},
$$

and

$$
\begin{aligned}
& r_{1}=\frac{\left(m_{0}-m_{1}\right) \Gamma(\alpha+q-1)}{\lambda m_{0} \Gamma(q)}, \quad r_{2}=\frac{\left(k_{0}-k_{1}\right)\left(m_{0}-m_{1}\right) \Gamma(\alpha+q)}{\lambda k_{1} m_{0} \Gamma(q)}, \\
& r_{3}=\frac{\left(k_{0}-k_{1}\right)}{k_{0}} r_{1} .
\end{aligned}
$$

Then $M>1, r_{0}>0,0<r_{3}<r_{1}$, and $r_{2}>0$ if (H0) holds. And $r_{2} b<r_{1} d, r_{3} a<r_{1} d$ if $0<a<$ $b<r_{0} d$.

Theorem 3.1 Suppose that (H0) holds, there exist constants $a, b, c, d$ such that $0<a<b<$ $\frac{k_{1}}{k_{0}} c \leq r_{0} d<d$, and $f \in C([0,1] \times[0,+\infty) \times \mathbb{R},[0,+\infty))$ satisfies the following conditions:

$(\mathrm{H} 1) \quad 0 \leq f(t, u, v) \leq \varphi_{p}\left(r_{1} d\right)$ for any $(t, u, v) \in[0,1] \times[0, M d] \times[0, d]$;

(H2) $f(t, u, v)>\varphi_{p}\left(r_{2} b\right)$ for any $(t, u, v) \in[0,1] \times[b, c] \times[0, d]$;

(H3) $0 \leq f(t, u, v) \leq \varphi_{p}\left(r_{3} a\right)$ for any $(t, u, v) \in[0,1] \times\left[\frac{k_{1}}{k_{0}} a, a\right] \times[0, d]$.

Then the boundary value problem (1) has at least three positive solutions $x_{1}, x_{2}, x_{3} \in \bar{P}(\beta ; d)$, and the solutions are increasing and convex on $[0,1]$. Moreover, for any $t \in[0,1]$,

$$
\begin{aligned}
& 0 \leq x_{i}^{\prime}(t)<d, \quad i=1,2,3 ; \quad x_{1}(t)>b ; \\
& \max _{t \in[0,1]} x_{2}(t)>a, \quad \text { with } \min _{t \in[0,1]} x_{2}(t)<b ; \quad \text { and } \quad x_{3}(t)<a .
\end{aligned}
$$

Proof Define the nonnegative continuous convex functionals $\beta, \rho$ and the nonnegative continuous functional $\psi$, the nonnegative continuous concave functional $\omega$ on the cone 
$P$ by

$$
\begin{aligned}
& \beta(x)=\max _{t \in[0,1]}\left|x^{\prime}(t)\right|, \quad \rho(x)=\max _{t \in[0,1]}|x(t)|, \\
& \psi(x)=\max _{t \in[0,1]}|x(t)|, \quad \text { and } \quad \omega(x)=\min _{t \in[0,1]}|x(t)| .
\end{aligned}
$$

Obviously, $\psi(k x)=k \psi(x)$ for any $k \in(0,1), x \in P$.

Because

$$
\rho(x)=\max _{t \in[0,1]}|x(t)| \leq \frac{k_{0}}{k_{0}-k_{1}} \max _{t \in[0,1]}\left|x^{\prime}(t)\right|=M \max _{t \in[0,1]}\left|x^{\prime}(t)\right|=M \beta(x) .
$$

Then

$$
\|x\|=\max \left\{\max _{t \in[0,1]}|x(t)|, \max _{t \in[0,1]}\left|x^{\prime}(t)\right|\right\}=\max \{\rho(x), \beta(x)\} \leq M \beta(x) \quad \text { for } x \in P .
$$

Following from the proof of Lemma 2.3, we can get that $T x \in P,(T x)(t) \geq 0, T x$ is increasing and convex on $[0,1]$.

For any $x \in \bar{P}(\beta ; d)$ implies that $x(t) \geq 0, \beta(x)=\max _{t \in[0,1]}\left|x^{\prime}(t)\right| \leq d$, and

$$
\rho(x)=\max _{t \in[0,1]}|x(t)| \leq M \max _{t \in[0,1]}\left|x^{\prime}(t)\right|=\beta(x) \leq M \beta(x) \leq M d
$$

By the condition (H1), we have $0 \leq f\left(t, x(t), x^{\prime}(t)\right) \leq \varphi_{p}\left(r_{1} d\right)$. Hence,

$$
\begin{aligned}
\beta(T x) & =\max _{t \in[0,1]}(T x)^{\prime}(t)=(T x)^{\prime}(1) \\
& =\frac{m_{0}}{m_{0}-m_{1}} \cdot \frac{\lambda}{\Gamma(\alpha-1)} \int_{0}^{1}(1-\tau)^{\alpha-2} \varphi_{q}\left(\int_{0}^{\tau} f\left(s, x(s), x^{\prime}(s)\right) \mathrm{d} s\right) \mathrm{d} \tau \\
& \leq \frac{m_{0}}{m_{0}-m_{1}} \cdot \frac{\lambda}{\Gamma(\alpha-1)} \cdot r_{1} d \cdot \int_{0}^{1}(1-\tau)^{\alpha-2} \tau^{q-1} \mathrm{~d} \tau \\
& =\frac{m_{0}}{m_{0}-m_{1}} \cdot \frac{\lambda \Gamma(q)}{\Gamma(\alpha+q-1)} \cdot r_{1} d \\
& =d .
\end{aligned}
$$

Therefore $T x \in \bar{P}(\beta ; d)$. By Lemma 2.3, T: $\bar{P}(\beta ; d) \rightarrow \bar{P}(\beta ; d)$ is a completely continuous operator.

$$
\begin{aligned}
& \text { Let } x_{0}=\frac{b+c}{2} \text {, then } \rho\left(x_{0}\right)=x_{0}=\frac{b+c}{2} \leq c, \beta\left(x_{0}\right)=0 \leq d \text {, and } \omega\left(x_{0}\right)=x_{0}=\frac{b+c}{2}>b \text {, so } \\
& x_{0} \in\{x \in P(\beta, \rho, \omega ; b, c, d) \mid \omega(x)>b\} \neq \phi .
\end{aligned}
$$

For any $x \in P(\beta, \rho, \omega ; b, c, d)$, it follows from the condition (H2), $f\left(t, x(t), x^{\prime}(t)\right)>\varphi_{p}\left(r_{2} b\right)$. By the proof of Lemma 2.3 and (7), we can get

$$
\begin{aligned}
\omega(T x) & =\min _{t \in[0,1]}|(T x)(t)|=(T x)(0) \\
& =\frac{m_{1}}{m_{0}-m_{1}} \cdot \frac{\lambda}{\Gamma(\alpha-1)} \cdot \frac{k_{1}}{k_{0}-k_{1}} \int_{0}^{1}(1-\tau)^{\alpha-2} \varphi_{q}\left(\int_{0}^{\tau} f\left(s, x(s), x^{\prime}(s)\right) \mathrm{d} s\right) \mathrm{d} \tau
\end{aligned}
$$




$$
\begin{aligned}
& +\frac{k_{1}}{k_{0}-k_{1}} \cdot \frac{\lambda}{\Gamma(\alpha)} \int_{0}^{1}(1-\tau)^{\alpha-1} \varphi_{q}\left(\int_{0}^{\tau} f\left(s, x(s), x^{\prime}(s)\right) \mathrm{d} s\right) \mathrm{d} \tau \\
> & \frac{m_{1}}{m_{0}-m_{1}} \cdot \frac{\lambda}{\Gamma(\alpha)} \cdot \frac{k_{1}}{k_{0}-k_{1}} \int_{0}^{1}(1-\tau)^{\alpha-1} \varphi_{q}\left(\int_{0}^{\tau} f\left(s, x(s), x^{\prime}(s)\right) \mathrm{d} s\right) \mathrm{d} \tau \\
& +\frac{k_{1}}{k_{0}-k_{1}} \cdot \frac{\lambda}{\Gamma(\alpha)} \int_{0}^{1}(1-\tau)^{\alpha-1} \varphi_{q}\left(\int_{0}^{\tau} f\left(s, x(s), x^{\prime}(s)\right) \mathrm{d} s\right) \mathrm{d} \tau \\
= & \frac{m_{0}}{m_{0}-m_{1}} \cdot \frac{k_{1}}{k_{0}-k_{1}} \cdot \frac{\lambda}{\Gamma(\alpha)} \int_{0}^{1}(1-\tau)^{\alpha-1} \varphi_{q}\left(\int_{0}^{\tau} f\left(s, x(s), x^{\prime}(s)\right) \mathrm{d} s\right) \mathrm{d} \tau \\
\geq & \frac{m_{0}}{m_{0}-m_{1}} \cdot \frac{k_{1}}{k_{0}-k_{1}} \cdot \frac{\lambda}{\Gamma(\alpha)} \cdot r_{2} b \cdot \int_{0}^{1}(1-\tau)^{\alpha-1} \tau^{q-1} \mathrm{~d} \tau \\
= & \frac{m_{0}}{m_{0}-m_{1}} \cdot \frac{k_{1}}{k_{1}-k_{0}} \cdot \frac{\lambda}{\Gamma(\alpha)} \cdot \frac{\Gamma(\alpha) \Gamma(q)}{\Gamma(\alpha+q)} r_{2} b \\
= & b .
\end{aligned}
$$

Thus, the condition (A1) in the Avery-Peterson theorem is satisfied.

For any $x \in P(\beta, \omega ; b, d)$ with $\rho(T x)>c$, i.e., $\rho(T x)=\max _{t \in[0,1]}|(T x)(t)|>c$. Then

$$
\omega(T x)=\min _{t \in[0,1]}|(T x)(t)| \geq \frac{k_{1}}{k_{0}} \max _{t \in[0,1]}|(T x)(t)|>\frac{k_{1}}{k_{0}} c>b .
$$

Consequently, the condition (A2) in the Avery-Peterson theorem is satisfied. It is clear that $\theta=0 \notin R(\beta, \psi ; a, d)$.

For any $x \in R(\beta, \psi ; a, d)$ with $\psi(x)=a$, it implies that

$$
\beta(x)=\max _{t \in[0,1]}\left|x^{\prime}(t)\right| \leq d
$$

and

$$
0 \leq x(t) \leq \max _{t \in[0,1]} x(t)=\psi(x)=a .
$$

It is easy to get that

$$
x(t) \geq \min _{t \in[0,1]} x(t) \geq \frac{k_{1}}{k_{0}} \max _{t \in[0,1]} x(t)=\frac{k_{1}}{k_{0}} a .
$$

It follows from the condition (H3) that

$$
0 \leq f\left(t, x(t), x^{\prime}(t)\right) \leq \varphi_{p}\left(r_{3} a\right),
$$

then we have

$$
\begin{aligned}
\psi(T x)= & \max _{t \in[0,1]}|(T x)(t)|=(T x)(1) \\
= & \frac{m_{1}}{m_{0}-m_{1}} \cdot \frac{k_{0}}{k_{0}-k_{1}} \cdot \frac{\lambda}{\Gamma(\alpha-1)} \int_{0}^{1}(1-\tau)^{\alpha-2} \varphi_{q}\left(\int_{0}^{\tau} f\left(s, x(s), x^{\prime}(s)\right) \mathrm{d} s\right) \mathrm{d} \tau \\
& +\frac{k_{0}}{k_{0}-k_{1}} \cdot \frac{\lambda}{\Gamma(\alpha)} \int_{0}^{1}(1-\tau)^{\alpha-1} \varphi_{q}\left(\int_{0}^{\tau} f\left(s, x(s), x^{\prime}(s)\right) \mathrm{d} s\right) \mathrm{d} \tau
\end{aligned}
$$




$$
\begin{aligned}
& <\frac{k_{0}}{k_{0}-k_{1}} \cdot \frac{m_{0}}{m_{0}-m_{1}} \cdot \frac{\lambda}{\Gamma(\alpha-1)} \cdot r_{3} a \cdot \int_{0}^{1}(1-\tau)^{\alpha-2} \tau^{q-1} \mathrm{~d} \tau \\
& =\frac{k_{0}}{k_{0}-k_{1}} \cdot \frac{m_{0}}{m_{0}-m_{1}} \cdot \frac{\lambda \Gamma(q)}{\Gamma(\alpha+q-1)} \cdot r_{3} a \\
& =a .
\end{aligned}
$$

So, the condition (A3) in the Avery-Peterson theorem holds.

Therefore, the conditions in the Avery-Peterson theorem are satisfied, and we can obtain that there exist three positive fixed points $x_{1}, x_{2}, x_{3} \in \bar{P}(\beta ; d)$ for the operator $T$ corresponding to positive solutions to the discrete second-order boundary value problem (1) such that

$$
\begin{aligned}
& \beta\left(x_{i}\right)<d, \quad i=1,2,3 ; \quad \omega\left(x_{1}\right)>b ; \\
& \psi\left(x_{2}\right)>a, \quad \text { with } \omega\left(x_{2}\right)<b ; \quad \text { and } \quad \psi\left(x_{3}\right)<a .
\end{aligned}
$$

By Lemma 2.3, we can get that the solutions are increasing and convex on $[0,1]$. Hence

$$
\begin{aligned}
& 0 \leq x_{i}^{\prime}(t)<d, \quad i=1,2,3 ; \quad x_{1}(t)>b ; \\
& \max _{t \in[0,1]} x_{2}(t)>a, \quad \text { with } \min _{t \in[0,1]} x_{2}(t)<b ; \quad \text { and } \quad x_{3}(t)<a .
\end{aligned}
$$

\section{Example}

In this section, we give an example to illustrate Theorem 3.1.

Example 4.1 Consider the following Dirichlet-Neumann boundary value problem:

$$
\left\{\begin{array}{l}
\left(\left|{ }^{C} D^{\frac{7}{3}} x(t)\right|^{C} D^{\frac{7}{3}} x(t)\right)^{\prime}=f\left(t, x(t), x^{\prime}(t)\right), \quad t \in(0,1) \\
2 x(0)-x(1)=0 \\
3 x^{\prime}(0)-x^{\prime}(1)=0 \\
x^{\prime \prime}(0)=0, \quad x^{\prime \prime \prime}(0)=0
\end{array}\right.
$$

where

$$
f(t, u, v)=\left\{\begin{array}{l}
\frac{e^{t}}{10,000}+\frac{\left(35+34 u-36 u^{2}+72 u^{3}\right)\left(\Gamma\left(\frac{17}{6}\right)\right)^{2}}{135 \pi}+\frac{v}{20,000}, \quad u \in[0,1] \\
\frac{e^{t}}{10,000}+\frac{\left(-7,730+10,391 u-2,919 u^{2}+278 u^{3}\right)\left(\Gamma\left(\frac{17}{6}\right)\right)^{2}}{60 \pi}+\frac{v}{20,000}, \quad u \in[1,5] \\
\frac{e^{t}}{10,000}+\frac{\left(300+45 u-u^{2}\right)\left(\Gamma\left(\frac{17}{6}\right)\right)^{2}}{375,375 \pi}+\frac{v}{20,000}, \quad u \in[5,+\infty),
\end{array}\right.
$$

and

$p=3, \quad q=\frac{3}{2}, \quad \alpha=\frac{7}{3}, \quad k_{0}=2, \quad k_{1}=1$

$m_{0}=3, \quad m_{1}=1, \quad \lambda=1 \quad$ in the boundary value problem (1).

We choose $a=1, b=2, c=5, d=10$. We can easily get that $M=2>1, r_{0}=\frac{\Gamma\left(\frac{17}{6}\right)}{\Gamma\left(\frac{23}{6}\right)}, r_{1}=$ $\frac{4 \Gamma\left(\frac{17}{6}\right)}{3 \sqrt{\pi}}, r_{2}=\frac{4 \Gamma\left(\frac{23}{6}\right)}{3 \sqrt{\pi}}, r_{3}=\frac{2 \Gamma\left(\frac{17}{6}\right)}{3 \sqrt{\pi}}$.

After some calculation, we can check that $f(t, u, v)$ satisfies following conditions: 
(1) $0 \leq f(t, u, v)<\frac{160 \Gamma(17 / 6)^{2}}{\pi}+\frac{1}{1,000}<\varphi_{p}\left(r_{1} d\right)=\frac{1,600 \Gamma(17 / 6)^{2}}{9 \pi}$ for any

$(t, u, v) \in[0,1] \times[0, M d] \times[0, d]$

(2) $f(t, u, v)>\frac{60 \Gamma(17 / 6)^{2}}{\pi}+\frac{1}{1,000}>\varphi_{p}\left(r_{2} b\right)=\frac{64 \Gamma(23 / 6)^{2}}{9 \pi}$ for any

$(t, u, v) \in[0,1] \times[b, c] \times[0, d]$

(3) $0 \leq f(t, u, v)<\frac{4 \Gamma(17 / 6)^{2}}{9 \pi}+\frac{1}{1,000}<\varphi_{p}\left(r_{3} a\right)=\frac{\Gamma(17 / 6)^{2}}{\pi}$ for any

$(t, u, v) \in[0,1] \times\left[\frac{k_{1}}{k_{0}} a, a\right] \times[0, d]$.

Then all the conditions of Theorem 3.1 hold.

Hence, by Theorem 3.1, the boundary value problem (8) has at least three positive solutions $x_{1}, x_{2}, x_{3}$ such that

$$
\begin{aligned}
& 0 \leq x_{i}^{\prime}(t)<10, \quad i=1,2,3 \\
& x_{1}(t)>2 ; \quad \max _{t \in[0,1]} x_{2}(t)>1, \quad \text { with } \min _{t \in[0,1]} x_{2}(t)<2 ; \quad \text { and } \quad x_{3}(t)<1 .
\end{aligned}
$$

\section{Competing interests}

The authors declare that they have no competing interests.

\section{Authors' contributions}

All authors jointly worked on the results and they read and approved the final manuscript.

\section{Author details}

${ }^{1}$ College of Science, University of Shanghai for Science and Technology, Shanghai, 200093, China. ${ }^{2}$ Department of Mathematics, Beijing Institute of Technology, Beijing, 100081, China.

\section{Acknowledgements}

The authors wish to acknowledge the support by the Innovation Program of Shanghai Municipal Education Commission (No. 10ZZ93), and the National Natural Science Foundation of China (No. 11171220).

\section{Received: 17 February 2013 Accepted: 17 April 2013 Published: 2 May 2013}

\section{References}

1. Wang, J, Zhou, Y: A class of fractional evolution equations and optimal controls. Nonlinear Anal., Real World Appl. 12, 262-272 (2011)

2. Zhou, Y, Jiao, F: Existence of mild solutions for fractional neutral evolution equations. Comput. Math. Appl. 59, 1063-1077 (2010)

3. Wang, J, Zhou, Y, Wei, W, Xu, H: Nonlocal problems for fractional integrodifferential equations via fractional operators and optimal controls. Comput. Math. Appl. 62, 1427-1441 (2011)

4. Bai, Z: On positive solutions of a nonlocal fractional boundary value problem. Nonlinear Anal. 72, 916-924 (2010)

5. Kaufmann, E, Yao, KD: Existence of solutions for a nonlinear fractional order differential equation. Electron. J. Qual. Theory Differ. Equ. 71, 1-9 (2009)

6. Jia, M, Liu, X: Three nonnegative solutions for fractional differential equations with integral boundary conditions. Comput. Math. Appl. 62, 1405-1412 (2011)

7. Liu, X, Jia, M, Wu, B: Existence and uniqueness of solution for fractional differential equations with integral boundary conditions. Electron. J. Qual. Theory Differ. Equ. 69, 1-10 (2009)

8. $\mathrm{Xu}, \mathrm{Y}, \mathrm{He}, \mathrm{Z}$ : The short memory principle for solving Abel differential equation of fractional order. Comput. Math. Appl. 62, 4796-4805 (2011)

9. Agarwal, RP, Filippakis, M, O'Regan, D, Papageorgiou, NS: Twin positive solutions for $p$-Laplacian nonlinear Neumann problems via variational and degree theoretic methods. J. Nonlinear Convex Anal. 9, 1-23 (2008)

10. Cheng, Z, Ren, J: Periodic solutions for a fourth-order Rayleigh type $p$-Laplacian delay equation. Nonlinear Anal. 70, 516-523 (2009)

11. Du, Z, Lin, X, Tisdell, CC: A multiplicity result for $p$-Laplacian boundary value problems via critical points theorem. Appl. Math. Comput. 205, 231-237 (2008)

12. He, Z, Long, Z: Three positive solutions of three-point boundary value problems for $p$-Laplacian dynamic equations on time scales. Nonlinear Anal. 69, 569-578 (2008)

13. Yang, $X$, Wei, Z, Dong, W: Existence of positive solutions for the boundary value problem of nonlinear fractional differential equations. Commun. Nonlinear Sci. Numer. Simul. 17, 85-92 (2012)

14. Liu, X, Jia, M: Multiple solutions for fractional differential equations with nonlinear boundary conditions. Comput. Math. Appl. 59, 2880-2886 (2010)

15. Liu, Y: Positive solutions of mixed type multi-point non-homogeneous BVPs for $p$-Laplacian equations. Appl. Math. Comput. 206, 796-805 (2008) 
16. Liu, X, Jia, M, Xiang, X: On the solvability of a fractional differential equation model involving the $p$-Laplacian operator. Comput. Math. Appl. 62, 1405-1412 (2011)

17. Chen, T, Liu, W, Hu, Z: A boundary value problem for fractional differential equation with $p$-Laplacian operator at resonance. Nonlinear Anal. 75, 3210-3217 (2012)

18. Podlubny, I: Fractional Differential Equations. Mathematics in Science and Engineering, vol. 198. Academic Press, New York (1999)

19. Avery, Rl, Peterson, AC: Three positive fixed points of nonlinear operators on ordered Banach spaces. Comput. Math. Appl. 42, 313-322 (2001)

doi:10.1186/1687-1847-2013-126

Cite this article as: Liu et al.: Multiple solutions of a $p$-Laplacian model involving a fractional derivative. Advances in Difference Equations 2013 2013:126.

Submit your manuscript to a SpringerOpen ${ }^{\odot}$ journal and benefit from:

- Convenient online submission

- Rigorous peer review

- Immediate publication on acceptance

- Open access: articles freely available online

- High visibility within the field

- Retaining the copyright to your article

Submit your next manuscript at $>$ springeropen.com 